\title{
Controversias de la nueva ley educativa (LOMLOE): la concesión complementaria del título de la ESO en Formación Profesional Básica Controversies of the new educational law (LOMLOE): the complementary granting of the ESO degree in Basic Vocational Training
}

\author{
José Luis Martín Soria ${ }^{1}$, Paula Renés Arellano ${ }^{2}$, Ana Rosa Martín Minguijón ${ }^{3}$ \\ jmartin198@alumno.uned.es, paula.renes@unican.es, director@santander.uned.es \\ ${ }^{1}$ Doctorando programa Dcho. y C. \\ Sociales \\ UNED \\ España \\ ${ }^{2}$ Prof. ${ }^{\mathrm{a}}$ CD. dpto. $\mathrm{T}^{\mathrm{a}}$ e $\mathrm{H}^{\mathrm{a}}$ de la \\ Educación \\ Universidad Cantabria \\ España \\ ${ }^{3}$ Catedrática Dcho. Romano. Dir. ${ }^{\mathrm{a}}$ \\ UNED \\ Cantabria \\ España
}

\begin{abstract}
Resumen- Desde la llegada de la democracia a nuestro país con la Constitución de 1978, se han producido numerosos cambios estructurales en las políticas educativas, tantos como diferentes gobiernos hemos tenido en España. Estas reformas educativas, se orientaron principalmente a conseguir una educación de calidad para todas las personas, independientemente de sus características personales, económicas o sociales. Y la actual LOMLOE no es excepción. Una cuestión relevante que plantea esta ley, recientemente aprobada en el Congreso, es la concesión del título de ESO, para estudiantes que finalicen Formación Profesional Básica. Esta medida, orientada a atender a la diversidad facilitando la inclusión social, suscita controversias entre miembros de la comunidad educativa por considerarla desacertada. Indudablemente plantearía un atractivo para estudiantes al borde de la exclusión socioeducativa, para matricularse en FPB, especialmente alumnado con necesidades educativas especiales condenado a abandonar sin éxito su educación. Este estudio pretende aclarar el contexto normativo sobre la implementación de esta medida y fomenta el debate sobre si ¿es justa esta discriminación positiva comparada con otros programas educativos, o supone un agravio comparativo para los demás? Y también si ¿será esta medida verdaderamente útil y recomendable para su propósito, atender a la diversidad y facilitar la inclusión social?
\end{abstract}

\section{Palabras clave: Política educativa, LOMLOE, FPB, legislación educativa, inclusión social.}

\footnotetext{
Abstract- Since the arrival of democracy in our country with the 1978 Constitution, there have been numerous structural changes in educational policies, as many as different governments have had in Spain. These educational reforms were mainly aimed at achieving a quality education for all people, regardless of their personal, economic, or social characteristics. And the current LOMLOE is no exception. A relevant issue raised by this law, recently approved in Congress, is the granting of the ESO degree for students who complete FPB. This measure, aimed at addressing diversity by facilitating social inclusion, raises controversies among members of the educational community as it is considered inappropriate. Undoubtedly, it would pose an attraction for students on the verge of socio-educational exclusion, to enroll in FPB, especially students with special educational needs condemned to unsuccessfully abandon their education. This study aims to clarify the regulatory context on the implementation of this measure and encourages the debate on whether this positive discrimination is fair compared to other educational programs, or is it a comparative offense for others? And, if will this measure be truly useful and
}

recommendable for its purpose, attending to diversity and facilitating social inclusion?

Keywords: Education policy, LOMLOE, FPB, education legislation, social inclusion.

\section{INTRODUCCIÓN}

"La educación es una actividad radicalmente humana, sistemática, orientada al perfeccionamiento, a la mejora de las personas, de cada una de las personas, por medio de acciones intencionadas de los educadores, generalmente concretadas en planes o programas" (Pérez, 2006, p. 1).

Atender a la diversidad de cualquier persona, ofreciendo una educación de calidad para todos, se ha convertido en uno de los principales objetivos de las diferentes Administraciones educativas, que independientemente de su orientación política, han dirigido la educación en nuestro país en estos últimos años. Para facilitar esta cuestión, apostar por la inclusión socioeducativa, e intentar reducir el abandono y el fracaso escolar, suelen ser las principales líneas de actuación para responder a necesidades educativas, relacionadas con atender a la diversidad del alumnado. Por ello, los agentes responsables en materia educativa independientemente de su ideología política, suelen dirigir sus principales esfuerzos hacia estos aspectos con las propuestas de sus reformas pedagógicas que han puesto en práctica estos últimos años en nuestro país, Utilizar todos los mecanismos posibles para mantener la escolarización de personas que aun estando en la edad obligatoria para ello fracasan, se encuentren en situación de especial vulnerabilidad y/o exclusión social, e intentar por todos los medios coartar su posible abandono escolar, parece ser la pretensión fundamental.

Para este fin, una de las formas habituales de llevar a la práctica esta cuestión, está relacionada con la puesta en funcionamiento y desarrollo de proyectos educativos orientados a la atención a la diversidad y a la inclusión social de alumnado con necesidades educativas de algún tipo, destacando entre 
otros la Formación Profesional Básica, denominada así en el contexto actual. Pero no siempre se llamó así. Comenzó con el nombre de Garantía Social con la LOGSE. Posteriormente estos programas educativos se denominaron Programas de Cualificación Profesional Inicial, (PCPI) con la LOE. A continuación, Formación Profesional Básica, (FPB) en el nuevo contexto con la LOMCE, y en breve, con la LOMLOE se pasarán a denominar Ciclos Formativos de Grado Básico.

Es importante aclarar que, a su vez, la eficiencia de estos programas inclusivos está directamente relacionada con la educación en valores de los diferentes agentes implicados en la comunidad educativa que alberga a estos educandos, porque aunque sean estos, en definitiva, el objetivo principal de este esfuerzo orientado a la inclusión social, es precisamente la educación en valores de toda la comunidad educativa y en definitiva, de la sociedad que los acoge, la que sienta las bases estructurales para que el alumnado que participa en estos programas educativos, pueda llegar a lograr una plena y verdadera inclusión social. No sólo depende de que ellos cambien y se adapten a las normas socialmente impuestas en su contexto, sino de que sean también aceptados socialmente, en buena parte por los demás.

Podemos explicar los valores desde diversos enfoques cognitivos, si bien una de las acepciones más utilizadas desde su origen, podría estar relacionada con una perspectiva filosófica. En este contexto, tenemos la definición de Zubiri (1986), que ya hace más de tres décadas, y con plena vigencia todavía, concebía los valores como cualidades que nos permiten regular el mundo para vivir en él. Es decir, desde su punto de vista estos son medios que sirven para disciplinar la vida de las personas en el mundo. Y es evidente que, al organizar y marcar las pautas deseables de las personas en una comunidad, contribuyen a su vez a facilitar las relaciones sociales entre todos sus miembros y a crear por lo tanto un mundo mejor. Por ello, de aquí extraemos dos ideas importantes. Los valores como mecanismo estructural que sirve para organizar una vida en sociedad, y, por otro lado, como elementos que facilitan la cordialidad entre las relaciones sociales y por ello determinan que nuestra sociedad sea aún mejor. Podemos identificar por tanto que Zubiri relaciona en su definición, los valores con su utilidad social y que es fundamental educar en valores para poner en marcha los mecanismos que facilitan la inclusión social, y que deseamos para nuestros jóvenes, que tienen dificultades en su contexto escolar y social.

$\mathrm{Si}$ contextualizamos estas ideas el ámbito educativo, encontramos algunos programas inclusivos, como por ejemplo la Formación Profesional Básica que toman especial protagonismo para intentar facilitar la atención a la diversidad y la inclusión social de parte del alumnado que viene sufriendo habitualmente fracaso escolar durante sus años de escolarización. Situación ésta, que puede estar muchas veces asociada a otros problemas relacionados con un deficiente apoyo o influencia de su contexto social y/o familiar.

En esta línea, de la importancia del contexto familiar, indica Buxarrais, (2012):

La familia tiene un rol protagónico en la transmisión de valores ya que es la unidad clave en la configuración del sistema de valores de las personas. A pesar de los cambios estructurales y de contenido que está viviendo, sigue siendo el primer contexto del desarrollo humano y realización personal, $\mathrm{y}$ en tanto agrupamiento $\mathrm{y}$ organización tiene, sin duda, su supervivencia bien asegurada.

Se hace hincapié, por tanto, en la gran importancia que supone para los menores esta educación en valores en el contexto familiar, y suscita a la vez la siguiente cuestión, relacionada con los chicos y chicas que carecen de un contexto familiar adecuado o incluso desestructurado. ¿Reciben una íntegra educación en valores los jóvenes que no viven en un contexto familiar bien conformado? Y, por otro lado, ¿Qué consecuencias tiene un contexto familiar desestructurado en el desarrollo personal de un niño, en sus resultados académicos y en su inclusion social?

Los docentes que hemos participado en programas orientados a la inclusión social estamos habituados a encontrar la respuesta precisamente a esta cuestión entre el alumnado que cursa estos estudios y podemos identificar fácilmente la relación que existe entre los conceptos anteriormente planteados. Un contexto social y familiar desestructurado, afecta negativamente al desarrollo socioemocional del menor, a su educación en valores y a su rendimiento académico y lo avoca con bastante probabilidad, y en la mayoría de los casos, a la una situación de especial vulnerabilidad y posiblemente a la exclusión social.

Retomando aspectos relacionados con la política educativa, el actual Gobierno de España, ha impulsado una nueva reforma educativa, que se acaba de aprobar hace pocos meses en el Parlamento, consistente en la octava ley orgánica educativa, la LOMLOE, que plantea importantes modificaciones con respecto a las anteriores que la precedieron, LOMCE, LOE, LOGSE, etc. Entre las nuevas medidas que plantea este nuevo texto legislativo, que no deroga por completo a la anterior, sino que plantea numerosas modificaciones a ésta, propone una medida en concreto que afecta directamente al alumnado que cursa estudios en uno de estos programas educativos orientados a la inclusión social, en concreto la FPB. Esta propuesta consiste en la posibilidad de obtener la certificación en la ESO de modo complementario, a la titulación en el programa de FPB, a la vez y de modo obligatorio. Medida que estará totalmente garantizada para todo el alumnado que finalice $\mathrm{y}$ titule en estos estudios de formación profesional, sin ningún tipo de procedimiento evaluador intermedio, si bien es cierto que no será aplicada de modo inmediato.

Es precisamente esta cuestión, la que supone una importante novedad respecto del panorama legislativo anterior, y que a su vez suscita un cierto grado de polémica entre buena parte de los miembros de la comunidad educativa, que andan precisamente divididos a la hora de discernir entre la idoneidad y utilidad práctica derivada del efecto que causará esta decisión de modificación normativa, entre el alumnado potencialmente usuario del programa y el resto.

\section{CONTEXTO}

Los docentes que desempeñan su carrera professional como profesores y tutores de FPB, conocen de primera mano las dificultades que supone para el alumnado, seguir un ritmo educativo adecuado, cuando el contexto familiar y el social no 
facilitan estas pretensiones. De hecho, la influencia de un ambiente negativo o poco favorecedor, que persiste a lo largo del tiempo, pueden avocar a cualquier joven, casi con total seguridad, hacia el más profundo fracaso escolar. Y lo que es aún peor, esta situación, también afectará de modo negativo, casi con toda probabilidad, del mismo modo, a su ámbito socioafectivo y personal. En este sentido, un estudio realizado por Caro y otros, en 2011, sobre la relación de determinantes socioculturales y su rendimiento académico en alumnos de enseñanza Secundaria obligatoria, recoge conclusiones como el hecho de que los alumnos que viven en entornos socioculturales más favorables, obtienen mejores resultados académicos, lo cual está relacionado con una mayor tasa de abandono escolar en poblaciones con recursos económicos y culturales limitados. Y existen numerosos trabajos que igualmente avalan esta cuestión.

En este contexto profesional docente, relacionado con impartir clases en programas educativos orientados a la inclusión socioeducativa, como puede ser la FPB, se hace bastante fácil experimentar dificultades didácticas y de manejo del aula al encontrarse en ciertos apuros derivados de trabajar habitualmente con el alumnado que sufre fracaso escolar y exclusión social. Muchos de estos profesionales de la docencia se ven obligados a tirar la toalla y terminan abandonando su trabajo, motivado habitualmente por sufrir continuamente una sensación de falta de control de situaciones vividas en el aula, de sentirse impotentes para motivar a estos estudiantes o para controlar el desarrollo de las clases del modo recomendable. De hecho, en más ocasiones de las deseadas se llega a sentir sensación de rabia por sufrir algunas situaciones complejas y experimentar la imposibilidad de actuar o no saber realmente qué hacer, ante los hechos acontecidos. Algunos de estos docentes llegan a tener incluso miedo por lo vivido en algunas circunstancias, y terminan padeciendo la peor sensación de todas que pueden experimentar un docente, y que a su vez supone consecuencias demoledoras para su alumnado. Terminar sufriendo desmotivación. Y no hay nada, más tóxico y destructivo para el aprendizaje de un estudiante, que tener un profesor sin motivación.

Ya de por sí, el alumnado que cursa estos programas educativos acostumbra a presentar bajos niveles de autoestima, derivados muchas veces de su continuo fracaso escolar, y una importante falta de motivación, lo que se materializa en numerosas muestras de desinterés hacia las clases impartidas por su profesor, conductas disruptivas en el aula, absentismo y estos aspectos, perpetúan de nuevo el fracaso escolar, y avocan de nuevo a muchos de estos estudiantes a la exclusión social. Sólo cabría añadir a este cóctel sinérgico de elementos nocivos el ingrediente estrella que sería un profesor desmotivado, para lograr una pócima de enorme capacidad destructiva, a su ya panorama educativo desolador.

Por todo ello, se hace necesario analizar los factores que afectan al proceso de enseñanza y aprendizaje de estos programas educativos, para poder plantear propuestas de mejora, especialmente aquellos cambios de carácter estructural, como podría ser la obtención complementaria del certificado de la ESO para los titulados en FPB, medida ésta, que en breve será efectiva, derivada de un cambio normativo, recogido en la actual LOMLOE. Pero habrá que discernir si esta modificación supone o no un verdadero factor positivo para esta cuestión. La inclusión socioeducativa de alumnado en situación de especial vulnerabilidad y/o exclusión social, susceptible de participar en estos programas educativos como la FPB, desde luego es necesaria, pero lo que no está tan claro es si el mecanismo de inclusión y socialización que se debe utilizar debe consistir en facilitar de modo excesivo esta doble certificación.

La presente investigación pretende servir de utilidad a los docentes que desempeñan su labor en estos programas, a estudiantes interesados en cursar estos estudios, a sus familias, a miembros de equipos directivos, así como de las Administraciones educativas $\mathrm{y}$, en definitiva, a toda la comunidad educativa en general, para entender mejor como afectarán los cambios normativos propuestos por la nueva Ley a esta cuestión.

\section{Para ello se plantean los siguientes objetivos:}

Conocer y entender el funcionamiento de los programas educativos de Formación Profesional Básica como facilitadores de la inclusión socioeducativa de alumnado en situación de especial vulnerabilidad y/o exclusión social.

Identificar las modificaciones normativas presentes en la LOMLOE, relacionadas con los programas orientados a la inclusión socioeducativa de alumnado con necesidades específicas de apoyo educativo, especialmente las relacionadas con la certificación y titulación obtenida por los estudiantes de estos programas de FPB al finalizar sus estudios.

Plantear las cuestiones susceptibles de ser formuladas, a partir del panorama educativo que se plantea en adelante con las modificaciones normativas que afectan a la posible doble titulación.

Abrir un debate constructivo en torno a esta cuestión para detectar posibles elementos susceptibles de mejora y si procede de modificación.

\section{DESCRIPCIÓN}

Fruto del análisis del marco teórico de la bibliografía y las publicaciones relacionada con la cuestión del estudio, así como la normativa directamente que afecta al estado de la cuestión, y la opinión derivada de entrevistas a varios docentes y expertos con el tema de investigación se ha extraído la información descrita en el presente ensayo, y entre otra la siguiente:

Desde hace ya algún tiempo, desde las administraciones educativas, se viene promoviendo el fomento entre el alumnado de la competencia social y cívica que viene definida como una de las competencias clave. Así desde la LOGSE, que a su vez venía recogiendo las orientaciones educativas propuestas a nivel europeo, ya venía definida ésta, como una de las principales competencias que debían fomentarse entre el alumnado en el contexto escolar. Y es por tanto, uno de los rasgos principales que deben caracterizar a un titulado en la ESO, tan importante como cualquier otra competencia, que se suelen identificar más facilmente de modo tradicional. En este aspecto, se hace fundamental la presencia de programas educativos que faciliten esta pretensión, especialmente para el alumnado que presenta ciertas dificultades de adaptación. Para ello, además, la educación en valores es una herramienta fundamental, para que los estudiantes, además de conocimientos curriculares, presenten sobre todo buenas cualidades en el plano personal y actitudinal. Y que, por desgracia, da la sensación de que últimamente se parece olvidar. 
Esta idea nos recuerda alguno de los preceptos del ensayo publicado en 1996, y reeditado en 2013, para la UNESCO por Jacques D'Lors, el que fue presidente de la Unión Europea. Se titulaba Los Cuatro Pilares de la Educación, y en aquel trabajo se hacía precisamente alusión a esta cuestión. Destacaba la necesidad de enseñar a nuestros jóvenes a aprender a vivir juntos y aprender a ser y señalaba estos aspectos como dos de los cuatro pilares fundamentales en los que se debe centrar precisamente la educación. Por tanto, a la intención de impartir enseñanza curricular, la escuela debe añadir la de educar en los valores sociales a nuestros niños y niñas, fomentar sus habilidades socioemocionales y garantizar que esta educación integral sirva para fomentar una buena convivencia con los demás.

Pero esta cuestión no es tan sencilla, en cierto modo porque se hace muy patente que cada vez vivimos en un mundo más caracterizado por la globalización y la diversidad. Y esto sucede en todo tipo de contextos. Político, religioso, económico, cultural, intelectual, etc. Por ello no es de extrañar que la escuela sea la primera institución que deba atender a esta diversidad, ofreciendo diferentes enfoques metodológicos que den respuesta a estas demandas tan diversas, y adaptando los diferentes proyectos educativos a cada una de ellas.

Haciendo de nuevo referencia a lo que se supone necesario para la obtención de un título de la ESO, identificamos por tanto que el estudiante debe haber alcanzado varias competencias definidas como clave, siendo una de ellas, tan importante como las otras, la definida como cívica y social. Y esto se logra, entre otros aspectos, con el trabajo día a día en el aula, aprendiendo valores además del contenido curricular.

Por ello, esta citada reforma educativa que se plantea con la presente LOMLOE, relacionada con la obtención automática del título de la ESO para el alumnado que finalice los estudios de FPB, y que será efectiva para los alumnos y alumnas que inicien estos estudios a partir del curso 2021/22, y finalicen en el curso 2023/24, no está exenta de cierta polémica entre muchos de los miembros de la comunidad educativa. Esto se deriva precisamente por las dificultades que encuentran estos docentes para lograr que sus educandos adquieran este tipo de competencias además de las curriculares. Nos referimos a las relacionadas con aspectos socioemocionales, cívicos y el ámbito ético y moral.

La controversia sucede, en buena parte, por la falta de consenso que ha existido, para variar, con la aprobación de una nueva reforma educativa y al decidir implantar esta medida por medio de una imposición unilateral del actual Gobierno Central. Aunque bien es cierto que la reforma sea aprobada vía ley orgánica, tal y cómo requiere el desarrollo normativo de un derecho fundamental constitucional como la educación, y que requiere la mayoría del Congreso, sí que supone de nuevo un fracaso, que esta decisión no sea fruto de un tan ansiado pacto por la educación. Acuerdo que viene siendo tan solicitado desde hace muchos años por los agentes sociales y educativos y que tampoco ha parecido ser posible en esta ocasión. Para variar. Por otro lado, tampoco termina de ser aceptado porque aún no están del todo claros sus posibles efectos en el panorama educativo actual, especialmente entre el alumnado que se vea afectado por esta medida al realizar estos estudios de FPB que le permitirán la obtención de una doble titulación. Además, supondrá entre otras cuestiones que, a su vez, el alumnado que cursa los estudios de ESO, en sus diferentes versiones, se pueda sentir involucrado en un agravio comparativo, frente a sus compañeros de FPB, ya que tan sólo obtendrán prácticamente en el mismo tiempo, y con similar o incluso mayor esfuerzo una única titulación. De hecho, existe una buena parte de miembros de la comunidad educativa, que independientemente de su ideología política, no ven con buenos ojos esta medida, y que no auguran buenos resultados para esta pretensión.

\section{Resultados}

Si concretamos los resultados del análisis de la norma educativa que se modifica y las consecuencias para el alumnado inscrito en el programa educativo de FPB, identificamos que la reforma que plantea la actual LOMLOE relacionada con la certificación en la ESO para los titulados en FPB, consiste en un aliciente muy atractivo, pero no exento de polémica, por varias cuestiones. Ahora bien, esto se justifica, en buena medida, con el siguiente argumento: Para un alumno que curse la FPB, suele darse la paradójica situación de ser un titulado en FPB y en cambio, no tener el certificado de la ESO, ya que lo habitual es que haya pasado de $2^{\circ}$ o $3^{\circ}$ de la ESO al primer curso de FPB. Y a la hora de lograr una inclusión socioeducativa efectiva y laboral, éste se hace un tanto compleja si no se acompaña de esta titulación en Educación Secundaria Obligatoria.

Ante esta casuística, se suscitó la cuestión, no exenta de opiniones contrapuestas y puntos de vista antagónicos, de que era conveniente facilitar algún procedimiento para que aquellos alumnos y alumnas que además de finalizar y titular en FPB, cuando alcanzasen ciertos objetivos o competencias similares a las que se alcanzan al finalizar la ESO, pudieran también titular con este certificado educativo. Para ello, en principio se aprobó una norma estatal que explicaba y desarrollaba esta opción educativa y su procedimiento. En concreto el Real Decreto $1058 / 2015$, de 20 de noviembre, en la disposición transitoria única establecía la posibilidad de que el alumnado titulado en Formación Profesional Básica en los cursos 2015/16 y 2016/17 pudiera obtener el título de Educación Secundaria Obligatoria. Pero estaba sometida a una serie de condiciones, que en muchos casos eran difíciles de cumplir.

Por ejemplo, los equipos docentes tenían que valorar si los alumnos/as habían alcanzado los objetivos de la Educación Secundaria Obligatoria y adquirido las competencias correspondientes a dicho currículo educativo. Además, en el caso de Cantabria, en las Instrucciones de Inicio de Curso, que consiste en un compendio de normas aclaratorias en las que se basa especialmente el desarrollo de cada año escolar, $y$ que la Consejería de Educación, Cultura y Deporte elaboró para ese curso, concretó el procedimiento que los centros habían de seguir en estos casos. En él se indicaba que para asegurar la coherencia en las decisiones que se adoptasen a este respecto, los equipos docentes, deberían tener fijados con anterioridad unos criterios consensuados que permitieran valorar si los alumnos/as cumplían las condiciones para la obtención del título de ESO.

Dichos criterios deberían ser valorados por todos los miembros del equipo docente y el profesorado de los ámbitos Sociolingüístico y Científico y Tecnológico. Estos deberían determinar, también en qué medida el alumno/a había alcanzado los objetivos establecidos para la ESO en el artículo 11 del Real Decreto 1105/2014, de 26 de diciembre, así como 
el grado de adquisición de las competencias, relacionadas con estos ámbitos.

En la práctica esta cuestión, se solucionó de varias formas, en función de las casuísticas y las decisiones tomadas en cada centro educativo, lo cual, determinaba que existieran ciertas diferencias de criterios de actuación, entre centros educativos. $\mathrm{Y}$ en definitiva que se aplicaran a una misma cuestión, diferentes requisitos en función de cada centro, lo cual no parecía demasiado coherente ni justo. Dependiendo del centro educativo los requerimientos exigidos para el logro de la doble certificación podían ser, y en muchos casos eran diferentes, lo cual determinada en algunos casos una situación de desigualdad, que no parecía coherente.

Algunas propuestas de decisiones tomadas a nivel de equipos docentes se asemejaban a las siguientes:

- Que, en los módulos de los ámbitos comunes, Sociolingüístico y Científico y Tecnológico, los alumnos/as obtuvieran como mínimo una calificación concreta, ya fuera $6,6,5,7$, etc.

- Con carácter general, que los alumnos/as no hubieran mostrado a lo largo del curso comportamientos disruptivos que influyeran negativamente en la convivencia, como por ejemplo que no hubieran protagonizado partes de conductas contrarias a la convivencia o expedientes disciplinarios.

\section{- Otras.}

Finalmente, la LOMCE, en su Disposición final quinta. En su Calendario de implantación, de nuevo retomaba esta problemática y lo regulaba expresamente en su apartado 4, y establecía de nuevo la misma consideración:

Hasta la entrada en vigor de la normativa resultante del Pacto de Estado social y político por la educación, los alumnos que obtengan un título de Formación Profesional Básica podrán obtener el título de Educación Secundaria Obligatoria, siempre que, en la evaluación final del ciclo formativo, el equipo docente considere que han alcanzado los objetivos de la Educación Secundaria Obligatoria y adquirido las competencias correspondientes.

En cambio, a partir de la LOMLOE, esto sucederá de modo automático, es decir, el alumnado que titule en FPB también lo hará en la ESO de modo complementario, sin necesidad de aprobación del equipo docente ni la exigencia de ningún requisito como sucedía en el panorama normativo anterior.

Así se expresa en el artículo 30 de la citada LOMLOE:

Artículo 30. Ciclos formativos de grado básico. 4. La superación de la totalidad de los ámbitos incluidos en un ciclo de grado básico conducirá a la obtención del título de Graduado en Educación Secundaria Obligatoria. Para favorecer la justificación en el ámbito laboral de las competencias.

$\mathrm{Si}$ bien es cierto que, la misma ley especifica en su disposición transitoria tercera, que define como Obtención del título de Educación Secundaria Obligatoria al superar la Formación Profesional Básica.

Hasta la implantación de las modificaciones introducidas en esta Ley relativas a la evaluación y titulación de los ciclos formativos básicos el alumnado que obtenga el título Profesional Básico podrá lograr el título de Graduado o Graduada en Educación Secundaria Obligatoria, siempre que haya superado las enseñanzas de los bloques y módulos en los que están organizados estos ciclos y el equipo docente considere que se han alcanzado los objetivos de educación secundaria obligatoria y adquirido las competencias correspondientes.

Con lo cual, aún quedan algunos años, en los que de momento la concesión del título de la ESO quedará sometido al criterio del equipo docente de los centros educativos, especialmente de sus profesores responsables de los ámbitos, y no será de modo obligatorio complementario hasta el curso 2023/2024 la concesión de este título complementario, según se dirime de la disposición final quinta, en su punto 6 de la citada ley, en la que se concreta su calendario de implantación, indicando lo siguiente:

\section{Calendario de implantación.}

6. Las modificaciones introducidas en el currículo, la organización y objetivos en los ciclos formativos de grado básico se implantarán en el curso que se inicie un año después de la entrada en vigor de esta Ley.

Con lo cual, si la entrada en vigor fue en enero del 2021, dicha modificación se aplicará en el curso que se inicie, en el 2022 , que sería el curso 2022/ 2023 y que finalizaría por tanto el 2023/24 con los primeros alumnos y alumnas doblemente titulados, tanto en FPB como en la ESO.

\section{CONCLUSIONES}

Una vez analizado el marco teórico relacionado con la cuestión de estudio, así como la normativa que afectará a los programas de FPB relacionada con su certificación complementaria con el título de la ESO, y motivada por la reforma educativa promovida por la LOMLOE, podemos destacar las siguientes conclusiones:

En primer lugar, el hecho de que, para garantizar el éxito de los programas educativos orientados a la inclusión social, como este de FPB, se hace patente una necesidad de educar previamente en valores tanto a los receptores de estos programas, como a los miembros del contexto que les rodea. Especialmente si queremos lograr una inclusión social verdaderamente efectiva. En este sentido, aún queda mucho trabajo por hacer, y siguen siendo necesarios muchos esfuerzos en todos los frentes posibles. Sin este trabajo previo, se hace muy difícil garantizar el éxito de estos programas educativos, independientemente de los esfuerzos realizados en el plano normativo. Este aspecto sería especialmente importante para un alumnado que va a recibir una titulación en la ESO complementaria a la de FPB, que garantiza entre otras la adquisición de una competencia cívica y social, que requiere, por tanto, una importante formación en valores y en habilidades socioemocionales. Para ello, el trabajo de éstas se deberá intensificar en el aula, de modo transversal a la formación curricular ordinaria.

En segundo lugar, es conveniente destacar que suele ser precisamente la adquisición de la competencia social y cívica por sus educandos, a la que deben prestar habitualmente más esfuerzo los docentes que trabajan en estos programas educativos. El hecho de facilitar una titulación complementaria en la ESO, por sí sola, no es garantía de la adquisición de las competencias que se supone acredita dicha certificación, como 
por ejemplo la citada competencia cívica y social. Esto suscita la polémica cuestión, entre otras, de si no sería conveniente plantear alguna exigencia o requisito complementario, para su obtención. Además, también es cierto que esta cuestión de educar en valores y trabajar habilidades socioemocionales se torna especialmente complejo para un solo docente en el aula, y con el alumnado de estos programas. Por ello, también se proponen entre otras medidas para la FPB dotar de apoyos de otros docentes en el aula $\mathrm{y}$ otros profesionales como especialistas en Pedagogía terapéutica, Servicios a la Comunidad o Educación Social, etc., que podrían reforzar enormemente esta adquisición de valores por parte del alumnado, atender de un modo más particular a sus necesidades, coartar posibles conductas disruptivas y en definitiva intensificar la calidad de estos programas.

Por otro lado, la medida propuesta respecto a la concesión de la doble titulación de los estudiantes en FPB, evidentemente supondrá un aliciente y un atractivo para los futuros participantes en estos programas educativos, pero igualmente podría plantear un agravio comparativo para los demás. Por ello, sería conveniente intensificar en la medida de lo posible, entre el alumnado de FPB, aquellos aspectos y competencias que se relacionan con la ESO, por ejemplo, introduciendo, contenidos y procedimientos que relacionen y acerquen un poco más ambas titulaciones. Por ejemplo, trabajar competencias exigidas para la ESO llevadas a cabo con procedimientos de trabajo y supuestos prácticos dentro cada temática y naturaleza del programa de FPB, en torno a una educación en valores de modo transversal, y el desarrollo de las habilidades socioemocionales del alumnado en todo momento.

Que, a pesar de no contar con el consenso de los agentes educativos, la medida ya ha sido aceptada, derivado de la aprobación de la ley orgánica educativa, si bien no entrará en vigor de modo inmediato y será aplicada para los estudiantes que finalicen los estudios a partir del curso 2023/24. Por ello, quizá sea el momento de investigar a cerca de esta cuestión, para intentar conocer la percepción de sus verdaderos protagonistas. Familias, docentes, equipos directivos, alumnado, reesponsables politicos, etc. De este modo podremos recabar más información sobre la idoneidad de esta medida, y plantear propuestas de mejora a las dificultades que presenta, con la mayor antelación possible a su aplicación.

Finalmente conviene destacar, que la medida propuesta, pese a no contar con el beneplácito de una buena parte de la comunidad educativa, especialmente docentes y responsables de estos programas educativos, se llevará a la práctica con relativa inmediatez, por ello, sería conveniente estudiar y analizar sobre los posibles resultados derivados de esta modificación normativa y plantear las actuaciones que surjan de estas consideraciones previas. Por ejemplo, podría ser interesante realizar un análisis comparativo de diferentes contextos territoriales sobre la percepción de la idoneidad de la medida propuesta entre diferentes miembros de la comunidad educativa. Incluso investigar sobre si existen precedentes en otros contextos internacionales y experiencias ya desarrolladas en este sentido, la percepción del alumnado susceptible de cursar estos estudios y cómo afecta a su motivación y su decisión, etc. El nuevo cambio legislativo que se implementará respecto a la $\mathrm{FPB}$, requiere de procesos de análisis $\mathrm{e}$ investigación. Por ejemplo, en torno a las necesidades que podrían aparecer de formación para los docentes que se enfrentarán a este reto, de las posibles adaptaciones curriculares que fueran necesarias para el alumnado de estos programas, de si esta medida ayudará o no a mejorar la competencia profesional de los estudiantes, su inclusión social, y de los retos y dificultades que se plantearán tras este nuevo cambio normativo.

\section{REFERENCIAS}

Buxarrais, M. R. (2012). Cómo viven las familias la educación en valores. Adolescencia, aprendizaje y personalidad. Barcelona: Sello Editorial.

Caro, L. G. C., Preciado, V. G., Pérez, L. M. L., Carrizosa, M. V., \& Molina, S. F. (2011). Determinantes socioculturales: su relación con el rendimiento académico en alumnos de Enseñanza Secundaria Obligatoria. Revista de investigación educativa, 29(1), 83-96.

Delors, J. (2013). Los cuatro pilares de la educación. Galileo, (23).

Ley Orgánica 1/1990, de 3 de octubre, de Ordenación General del Sistema Educativo.

Ley Orgánica 2/2006, de 3 de mayo, de Educación.

Ley Orgánica 8/2013, de 9 de diciembre, para la mejora de la calidad educativa.

Ley Orgánica 3/2020, de 29 de diciembre, por la que se modifica la Ley Orgánica 2/2006, de 3 de mayo, de Educación.

Pérez Juste, R. (2000). La evaluación de programas educativos: conceptos básicos, planteamientos generales $\mathrm{y}$ problemática.

Zubiri, X. (1986). Sobre el hombre. Madrid: Alianza Sociedad de Estudios y Publicaciones 